\title{
"Wearables on vogue": a scoping review on wearables on physical activity and sedentary behavior during COVID-19 pandemic
}

\author{
Rohit Muralidhar Panicker ${ }^{1} \cdot$ Baskaran Chandrasekaran $^{1}$ (D)
}

Received: 16 June 2021 / Accepted: 9 December 2021 / Published online: 7 January 2022

(c) The Author(s) 2022

\begin{abstract}
Background Wearables are intriguing way to promote physical activity and reduce sedentary behavior in populations with and without chronic diseases. However, the contemporary evidence demonstrating the effectiveness of wearables on physical health during the COVID-19 pandemic has yet to be explored.

Aim The present review aims to provide the readers with a broader knowledge of the impact of wearables on physical health during the pandemic.

Methods Five electronic databases (Web of Science, Scopus, Ovid Medline, Cumulative Index to Nursing and Allied Health Literature and Embase) were searched. The eligibility criteria of the studies to be included were based on PICOT criteria: population (adults, children and elderly), intervention (wearable, smartphones), comparison (any behavioral intervention), outcome (physical activity or sedentary behavior levels) and time frame (between December 1st, 2019 and November 19th, 2021). The present scoping review was framed as per the guidelines of the Arksey and O'Malley framework.

Results Of 469 citations initially screened, 17 articles were deemed eligible for inclusion and potential scoping was done. Smartphone-based applications with inbuilt accelerometers were commonly used, while a few studies employed smart bands, smartwatches for physical health monitoring. Most of the studies observed the increased use of wearables in healthy adults followed by elderly, children and pregnant women. Considerable reduction (almost-50\%) in physical activity during the pandemic: daily step count (-2812 steps/min), standing (-32.7\%) and walking $(-52.2 \%)$ time was found.

Conclusion Wearables appears to be impending means of improving physical activity and reducing sedentary behavior remotely during the COVID-19 pandemic.
\end{abstract}

Keywords Physical activity $\cdot$ Wearable $\cdot$ Smartwatch $\cdot$ Sedentary behavior $\cdot$ COVID-19 $\cdot$ Lockdown

\section{Introduction}

COVID-19 has emerged as a public health emergency affecting millions of people's physical, psychological, and social well-being [1]. State and national governments have enforced lockdowns, home confinements, restrictions on social gatherings and a ban on sports or exercise amenities to contain the spread of the virus [2]. However, the above protective measures have adversely affected the physical and mental health of the global population. Further remote work and virtual classrooms have intensified the physical

Baskaran Chandrasekaran

baskaran.c@manipal.edu

1 Department of Exercise and Sports Sciences, Manipal College of Health Professions, Manipal Academy of Higher

Education, Manipal, Karnataka 576104, India inactivity and sedentary behavior in the community [3]. Physical inactivity is the inability to meet the global recommendations of 150-300 min of moderate-to-vigorous exercise per week, while sedentary behavior is defined as any waking activity that is characterized by a low energy expenditure (i.e., less than 1.5 METS) [4]. Individuals with high physical inactivity and sedentary behavior are found to have a greater risk of chronic cardiometabolic diseases such as ischemic heart diseases, cancer, obesity, and early mortality $[5,6]$. Growing evidence states that physical inactivity and sedentary behavior have substantially increased during the pandemic due to work from home and high screen time [7, 8]. Contemporary evidence suggests that high sedentary time is associated with increased cardiometabolic disease risk independent of weekly physical activity time. Hence, lifestyle interventions focusing on reducing physical 
inactivity and sedentary behavior are perceived as intriguing measures to prevent the chronic disease risk.

\section{Wearables in promoting physical activity and reducing sedentary behavior}

A wearable is defined as "any body-worn computer that provides useful services while the user performs other tasks", and includes pedometers, smartwatches, smart wears and activity trackers [9]. The growing popularity of the Internet of Things and technologically sound users have raised the market value of wearables by USD 32.63 billion, and compound annual growth is projected as $15 \%$ from 2020 to 2027 [10]. By real-time visualizing, measuring and addressing sedentary behavior and physical activity bouts, wearables continue to increase in popularity and accessibility to the extensive data at the population level [11]. Difficulties in the raw data collection and transformation based on 'phantom' algorithms make commercial fitness trackers less reliable and valid than research-based accelerometers [12]. Despite its moderate accuracy, consumer-based wearables continue to be widely used to encourage physical activity and reduce sedentary behavior through behavior change techniques such as goal setting, prompts, cues, self-efficacy and social support [13].

Though wearables use became inevitable in contemporary men and women, the advocacy of wearables for promoting PA and reducing sedentary behavior during these unprecedented times is relatively unknown. Our aim was to provide a comprehensive overview of the breadth and results of studies examining the impact and extent of wearable use on the measurement, encouragement and monitoring of physical activity and sedentary behavior in individuals during successive lockdowns. We aimed to describe the wearables used in the promotion of physical activity (sensors to smartphone technology) as an initial contribution to the informative implementation in practice. We conducted a scoping review with the following objectives:

- To investigate the extent to which the wearables are being used to promote physical activity in the community

- To collate the evidence regarding the prevalence of physical inactivity and sedentary time in individuals measured and monitored using wearables.

\section{Methods}

The present scoping review followed the guidelines of Arksey and O'Malley framework, which consists of five mandatory stages: (1) identifying the research questions; (2) identifying relevant studies; (3) study selection; (4) charting the data; (5) collating, summarizing, and reporting the results
$[14,15]$. Scoping reviews share similar characteristics of systematic review except for the provision of a broader overview rather than answering a focused question.

\section{Identifying the research question}

Our present scoping review was initiated with a question "What is the breadth of wearable use, its physical and social impact on people during an ongoing pandemic?" The present scoping review aimed to collate and summarize the contemporary evidence investigating the use of the wearables in promoting physical activity and the barriers during the COVID pandemic.

\section{Identifying the relevant studies}

We searched five electronic databases (Web of Science, Scopus, Cumulative Index to Nursing and Allied Health Literature, Ovid Medline and Embase) for the studies investigating wearables and their use to reduce sedentary behavior and improve PA in healthy and diseased populations during COVID-19 pandemic. We searched with the possible combinations of the MeSH terms "wearable activity trackers", "wearable activity monitors", "activity monitors", "activity trackers", "fitness trackers", "wearable fitness devices", "wearable technology", "wearable devices", "Fitbit", "physical activity", "physical exercise" "aerobic exercise", "sedentary behaviour", "sedentary time", "sedentary lifestyle", "physical inactivity" with several combinations of Boolean operators and wildcards. We limited our search to a time frame of December 1st, 2019, to May 10th, 2021. The search was again updated on November 19th, 2021. The sample search strategy is provided as an additional file.

\section{Study selection}

We included studies that have: (1) advocated any wearable or digital device; (2) investigated physical activity or sedentary behavior; (3) included only adults; (4) both healthy and diseased; (5) conducted within the timeframe (from December 1st, 2019, till May 10th, 2021. However, the search was again updated on November 19th, 2021); (6) both experimental and observational studies and (7) published only in English. The study selection was based on the PICOT framework, as presented in Table 1.

\section{Charting the data}

We extracted the following variables to a bespoke extraction sheet: author, year, country, design, the objective of the study, type of the wearable, physical activity and sedentary behavior (step count, step time, sitting time, and moderate-to-vigorous activity time, standing time, 
Table 1 Eligibility criteria by which potential studies included based on the PICOT criteria

\begin{tabular}{ll}
\hline Variable & Eligibility criteria for the studies to be included \\
\hline Population (P) & $\begin{array}{c}\text { Adults with or without chronic diseases in whom the wearables were employed to assess change in sedentary behaviour or } \\
\text { physical activity }\end{array}$ \\
Intervention (I) & $\begin{array}{c}\text { Studies should have specifically advocated or observed the effects of wearable devices (Fitbit, Polar global positioning system, } \\
\text { smart bands such as honor, Huawei, and smart wear) }\end{array}$ \\
Comparator (C) & $\begin{array}{l}\text { Control group with or without standardized monitors such as pedometers and accelerometers } \\
\text { Outcomes (O) }\end{array} \quad \begin{array}{l}\text { Step count, step count, sitting time, moderate-to-vigorous physical activity-measured through subjective or objective means } \\
\text { Time frame (T) }\end{array} \quad$\begin{tabular}{l} 
From December 1st, 2019, till May 10th 2021. Updated again at November 19th 2021 \\
\hline
\end{tabular}
\end{tabular}

calorie expenditure). The measurement errors and the authors attempt for appropriate correction were extracted. Furthermore, the sociodemographic influence on the measurement, if any, was recorded.

\section{Collating, summarizing, and reporting the results}

The evidence of the wearables and the associated physical, social and physiological effects were analyzed as qualitative analysis and synthesized narratively to provide the readers and policymakers with the broader knowledge of wearables and their physical, social and mental impact during the pandemic. The data extracted from the search results are provided in Table 2.

\section{Results}

Of 347 screened, seventeen articles were deemed eligible for inclusion and potential scoping was done. Figure 1 shows the flow of the screening and inclusion of the studies for the review. The majority of the studies $(n=14 ; 82 \%)$ included adult participants $(n=7,59,979)$ ranged from 10 to 742,000 . Heterogeneity in the study types was observed as follows: cross-sectional $(n=9 ; 53 \%)$, longitudinal $(n=4 ; 24 \%)$, retrospective $(n=1 ; 6 \%)$, narrative review $(n=1 ; 6 \%)$ and pilot non-randomized studies $(n=2 ; 12 \%)$. Similar heterogeneity was found in participants too in the included studies: pregnant women [27], elderly [24, 26], office workers [21], diabetes [18] and children [25]. All the studies were from high-income countries.

\section{Wearables and the measurement of physical and physiological variables}

Seven studies administered smartphone-based physical activity measurement through inbuilt accelerometers from which the captured data were transferred to the cloud server and visualized in smartphone applications [16-19, 21, 24, 31]. Majority of studies $(n=9 ; 53 \%)$ employed wrist bands and wristwatches of multiple technology firms (Apple,
Samsung, Xiaomi) and wearable research-based accelerometers [20, 23, 25, 26, 28-30, 32]. A few studies $(n=4$; $24 \%$ ) reported the psychometrics of the wearables, and lowto-moderate validity was found [21, 22, 27].

\section{Physical health measurement through wearables}

The mean follow-up period of longitudinal studies was 21 weeks [18, 20, 21, 24, 26, 27]. The average reduction in the daily step count after the pandemic compared to before the pandemic was 2812 steps/day. The studies had reported a significant reduction in standing time $(-32.7 \%)$, walking time $(-52.2 \%)$ and step count $(-29 \%)$ during lockdown when the physical activity was monitored with the wearables $[22,26]$. Mean energy expenditure was reduced to $70 \mathrm{kcal}$ compared to before pandemic [23]. Only one study by Vile et al. [27] reported a reduction in heart rate variability variable, especially high LF/HF ratio. Further reduction in step count and sleep time was found to be positively associated with body mass [19], depression [26] and workplace stress [32]. Various behavior change techniques such as self-efficacy, goal setting, prompt/cues, information and social networking were associated with the compliance of wearable use [24, 25, 31].

\section{Discussion}

From the evidence included, we found a significant increase in the use of wearables to improve physical activity during the confinement or lockdown periods [21, 24]. There is a convincing evidence to show that there is a significant decrease in physical activity and increased sedentary time in people around the world when judged objectively using wearables [16-32]. Figure 2 represents the summary of our findings. In the following sections, we discuss about: (1) the increased usage of the wearables in spite of the validity and reliability of the phantom algorithms; (2) role of wearables in regulating physical health during the lockdown. 


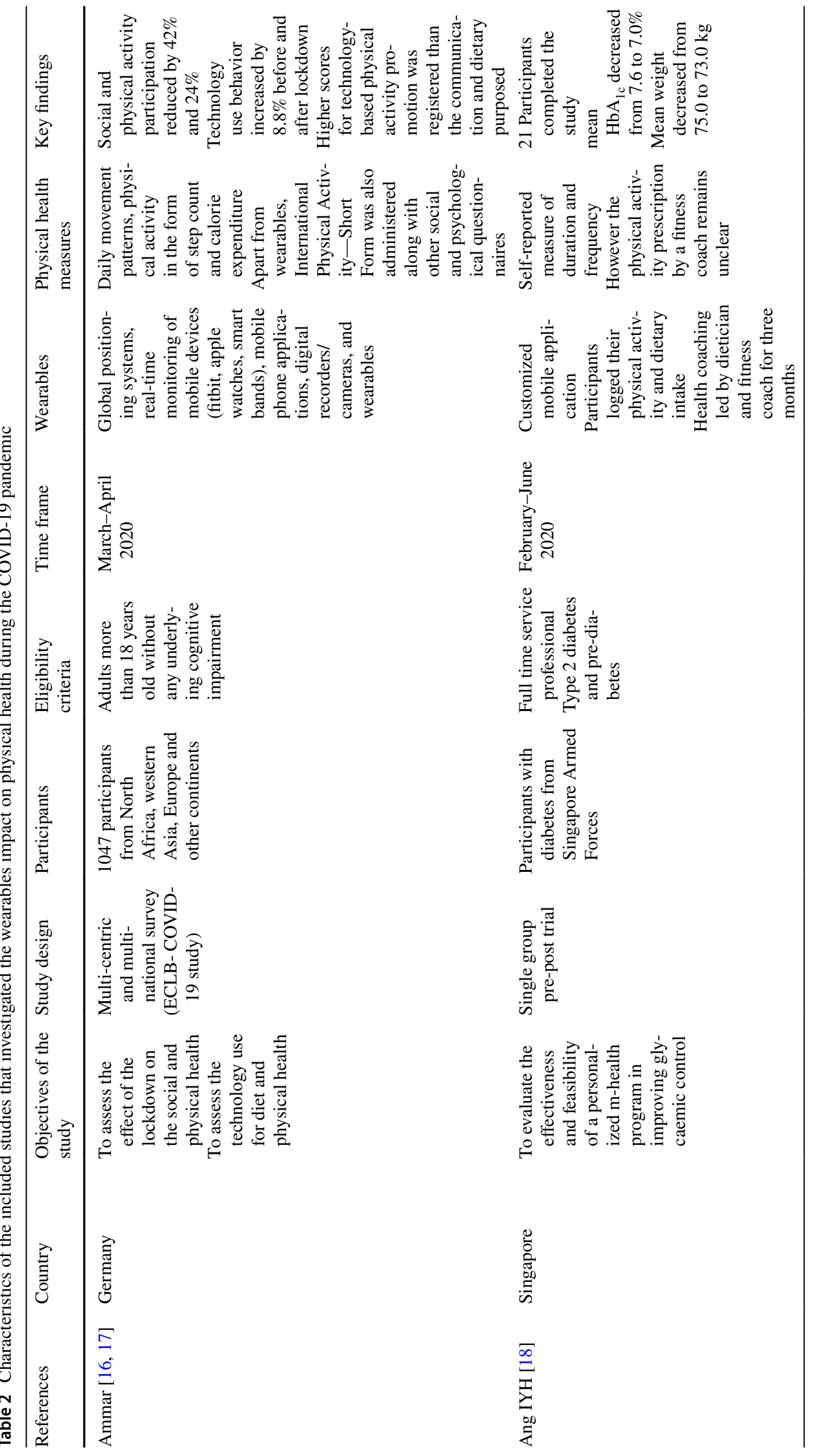


Sport Sciences for Health (2022) 18:641-657

645

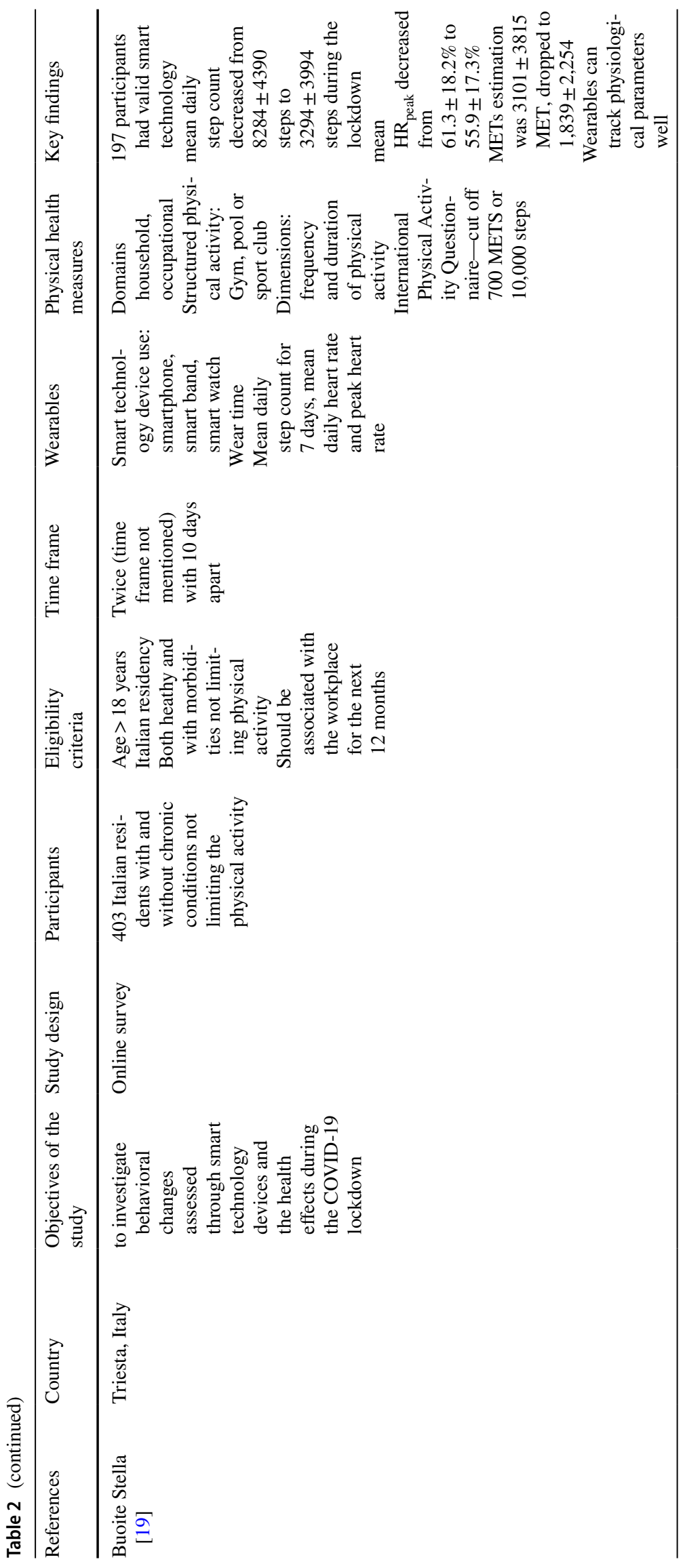

Springer 


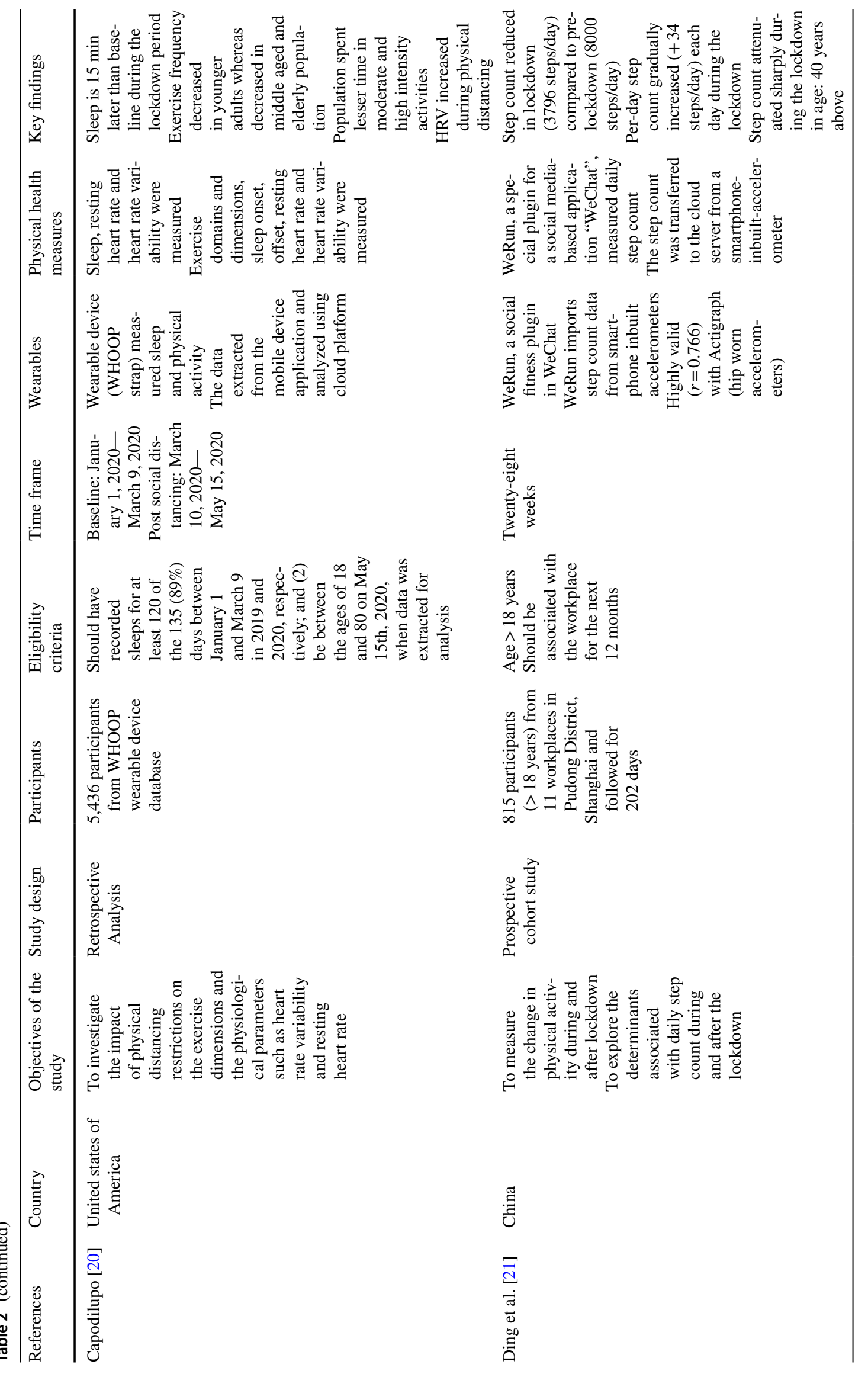




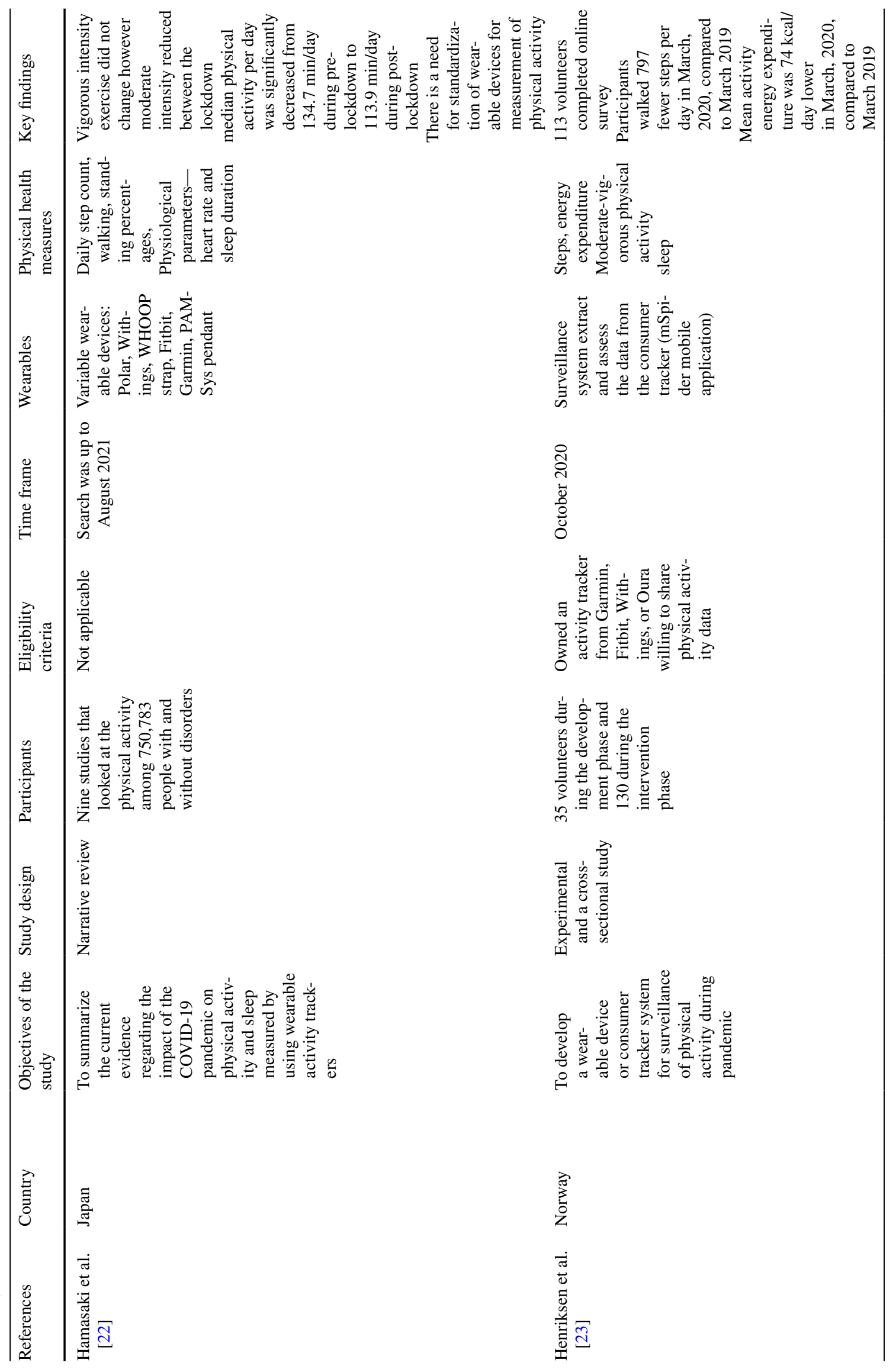




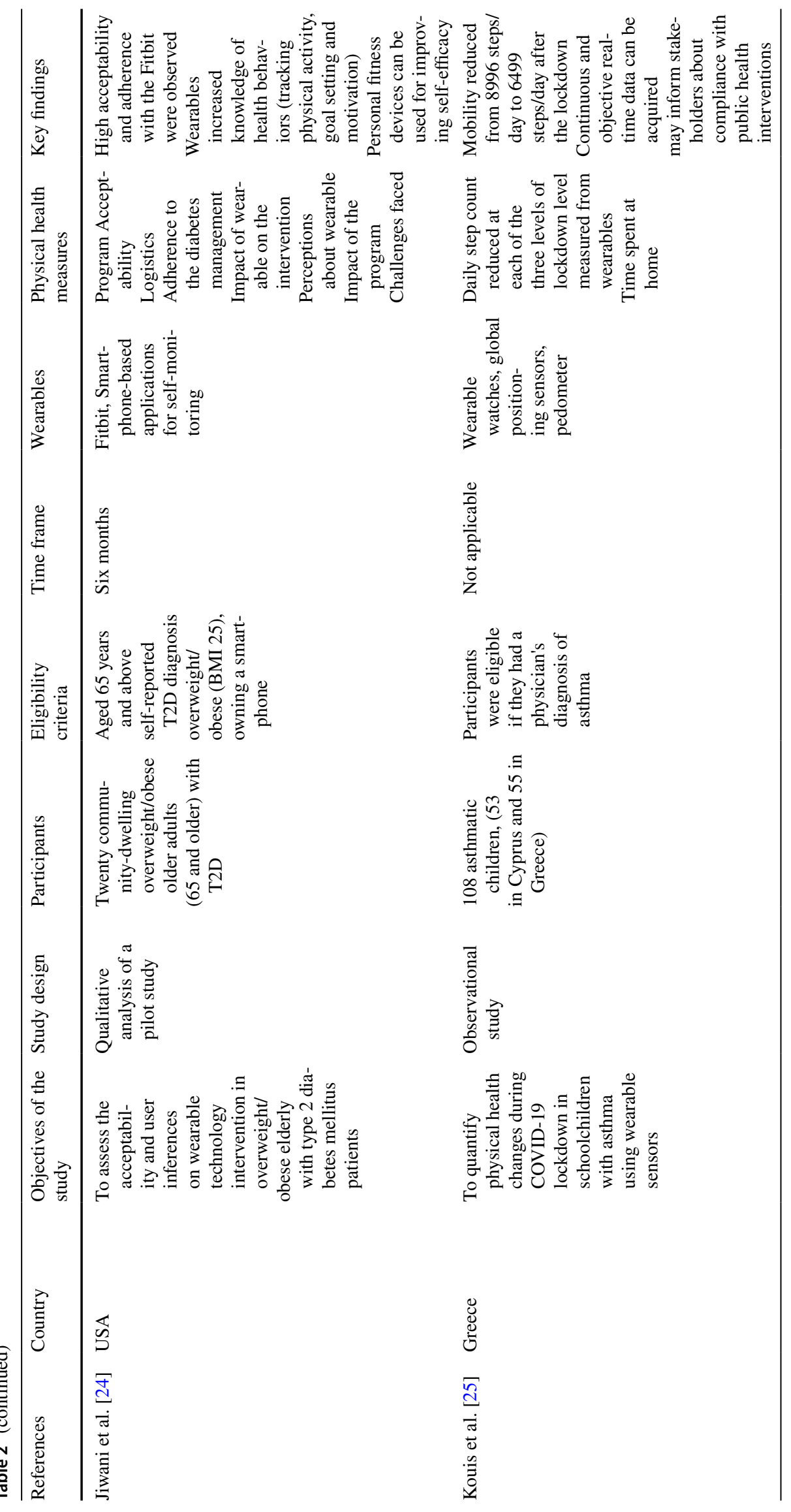




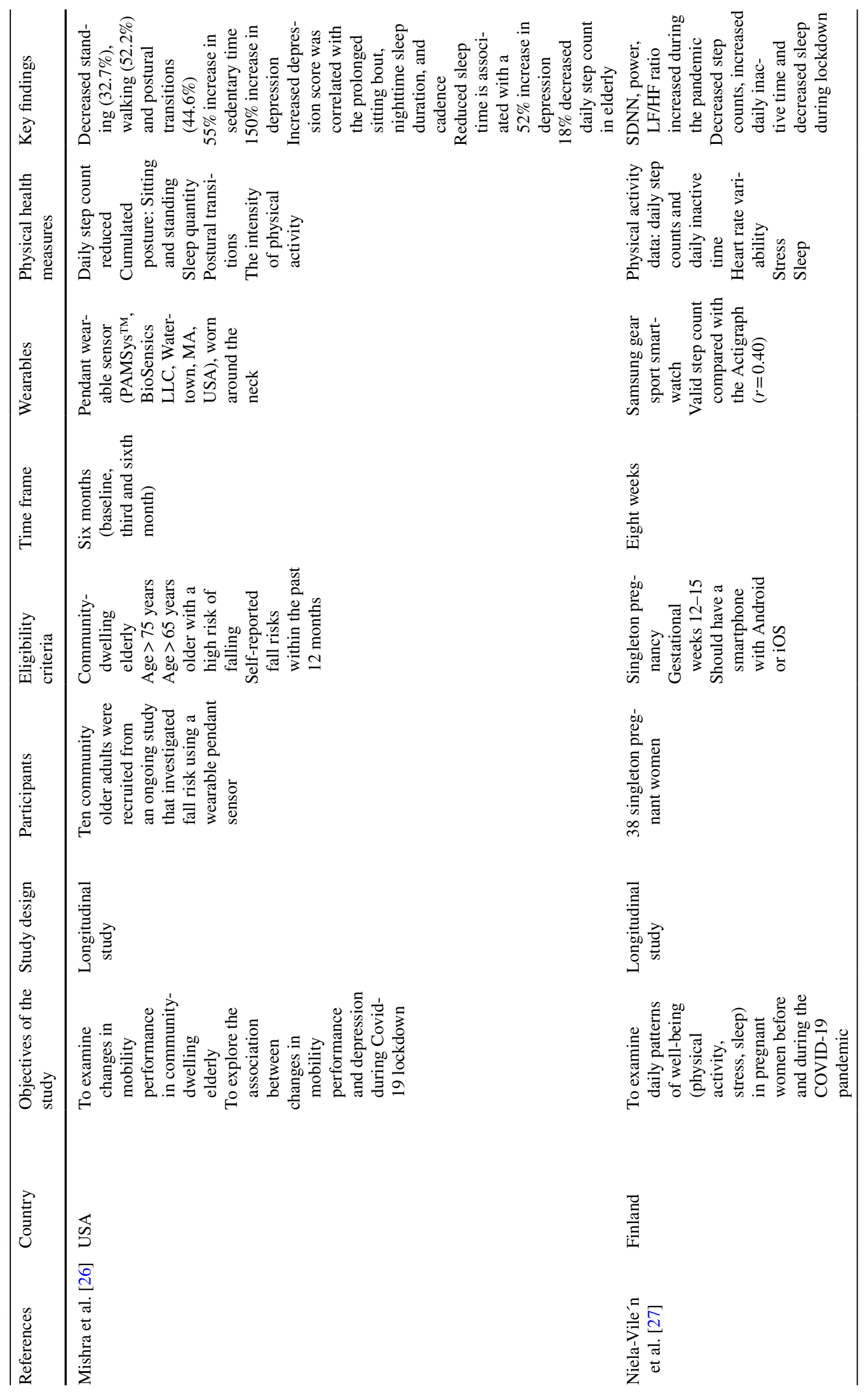




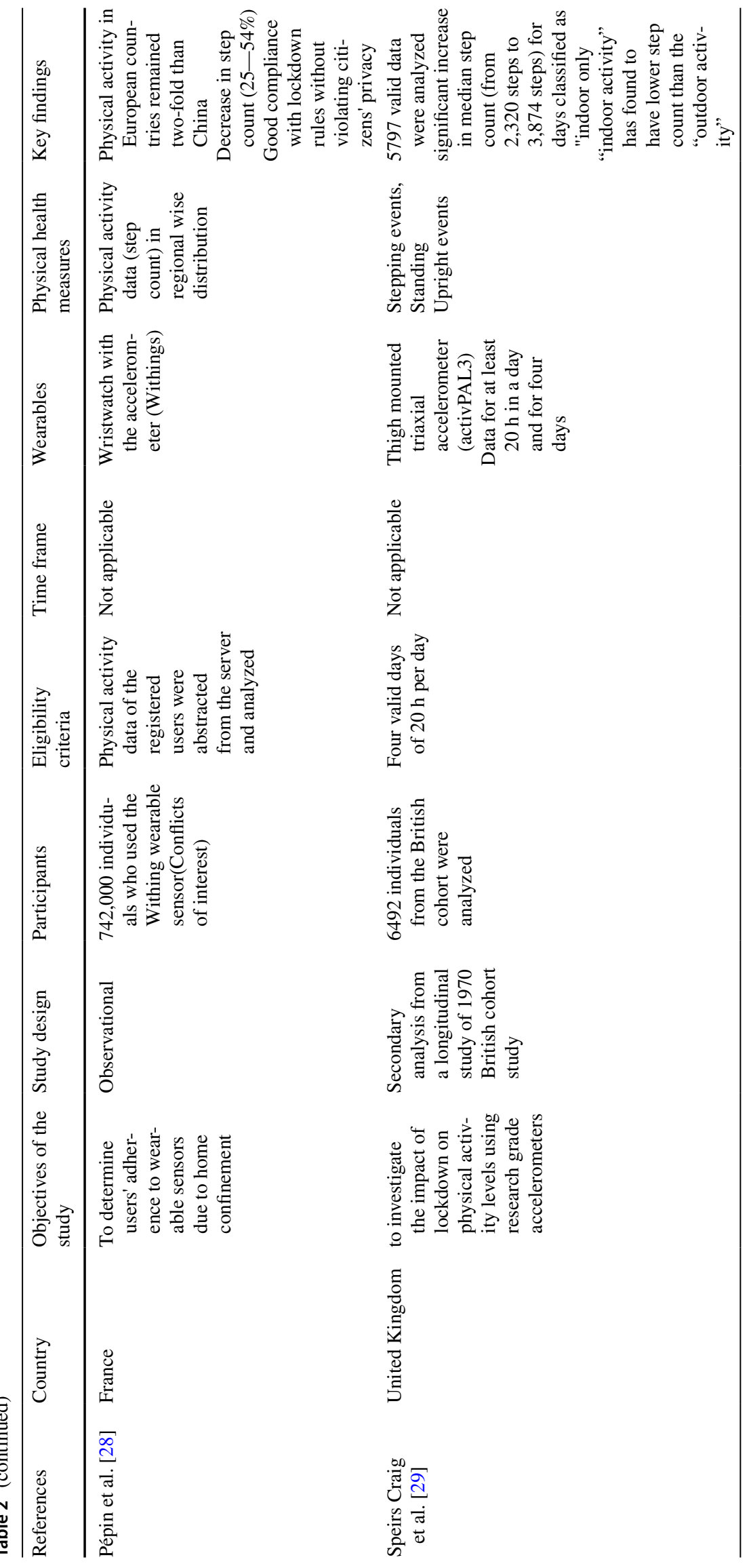




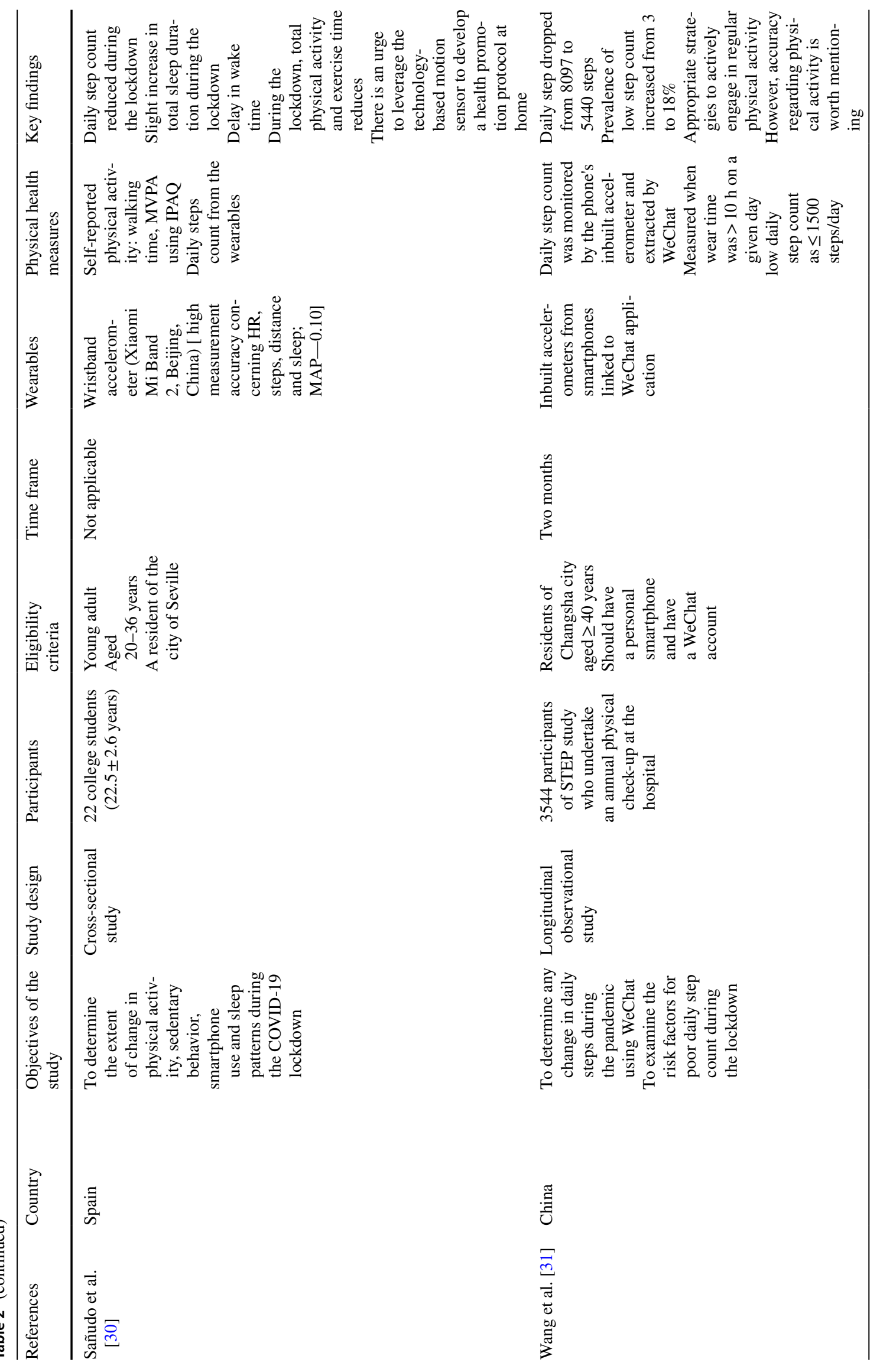




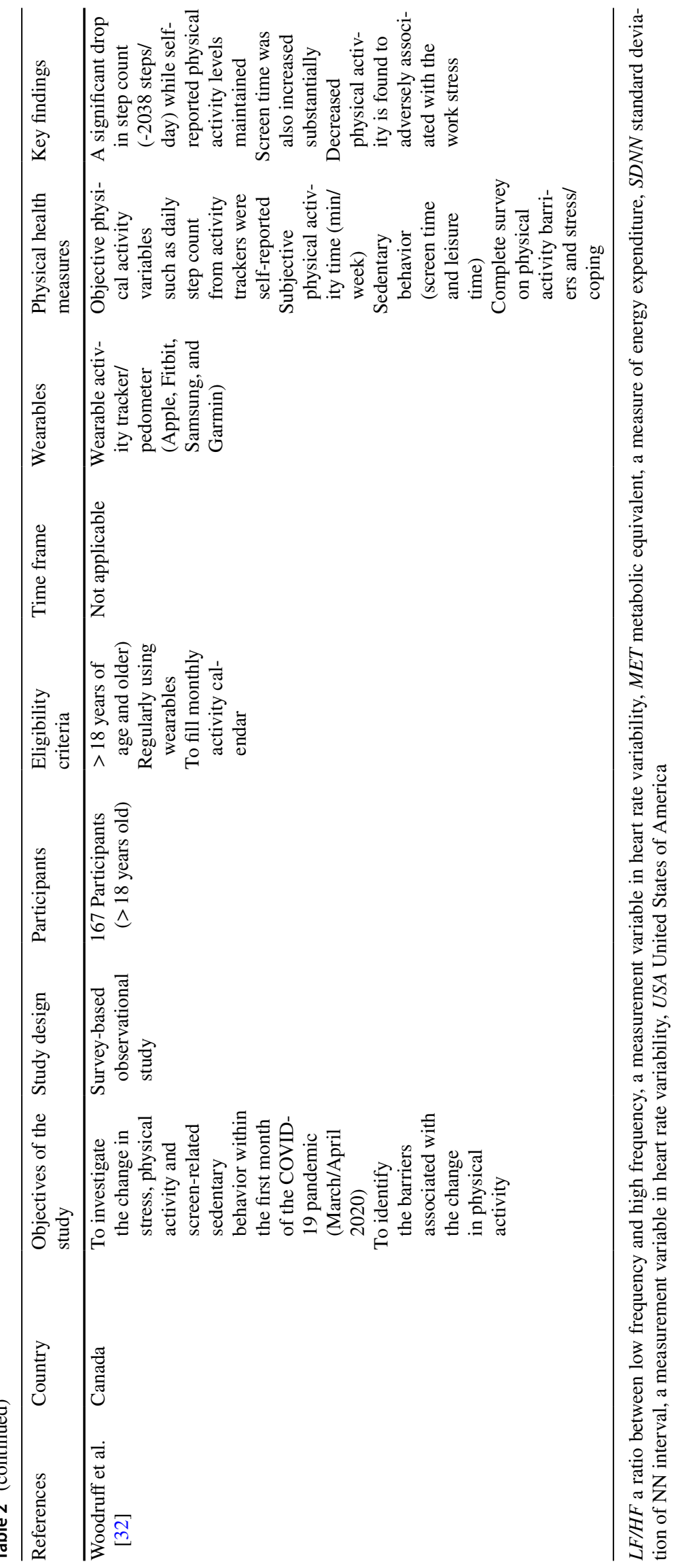



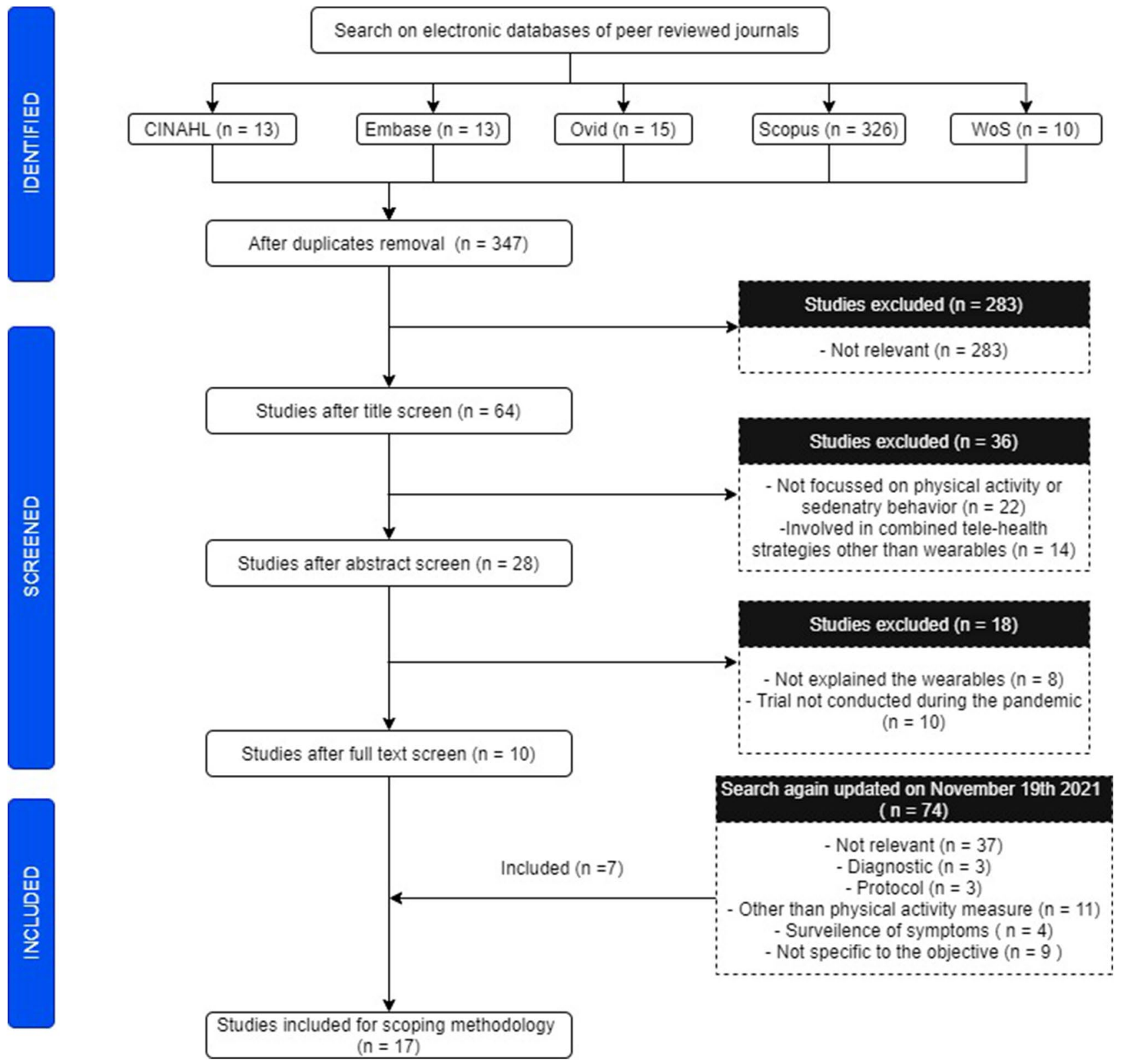

Fig. 1 Flowchart of the potential studies screened and included in the review which explored the wearables use for physical activity promotion and sedentary behavior reduction during the pandemic

\section{Use of wearables during COVID-19}

Wearables are intriguing means for measuring and monitoring physical activity at the population level in spite of arguments over their validity and reliability [22]. Cloud computing and artificial intelligence have leveraged healthcare through wearable sensors, increasing health monitoring and medical automation for speedy diagnosis, including COVID-19 [33]. The above facts are reciprocated with double-fold increase in utilization of wearable devices to self-monitor physical behavior during this pandemic [16-28,
34]. Although found to be less valid and reliable compared to research-based accelerometers, these wearables provide an opportunity for its end users to self-monitor their physical activity levels, energy expenditure, sleep, sitting time and create a framework for personalized prevention [34]. Our review findings also concur with the above findings from the largest British cohort study, the UK Biobank study [34]. Modern trends in mobile technologies have improved activity recognition and estimation of models making smartphones easily accessible and high accuracy in self-monitoring [35]. Recent wearables and their mobile application 
Fig. 2 Summary of the scoping review findings

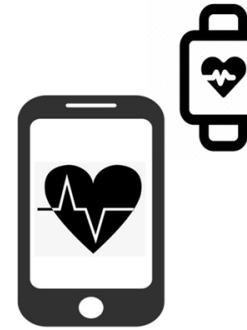

Wearables

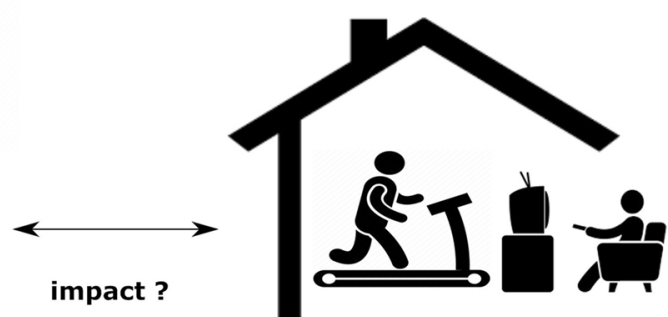

Physical health in lockdown

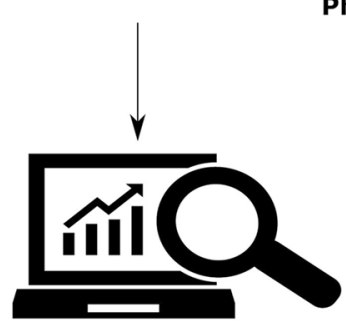

Five electronic databases

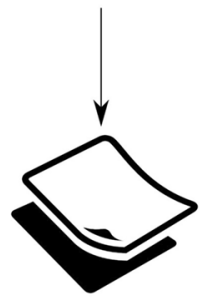

Seventeen research studies

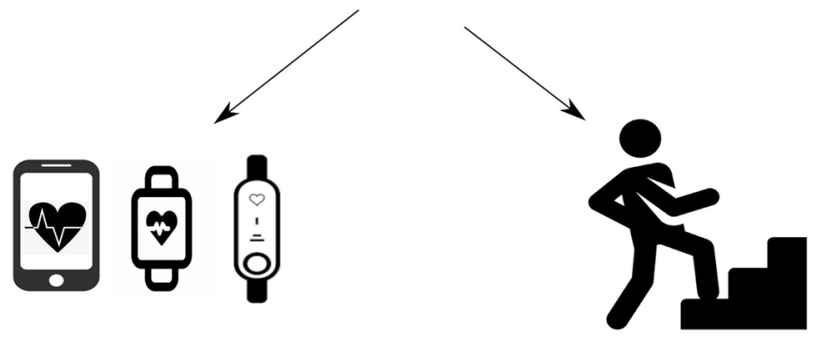

- Substantial increase in wearables

- Use of smartphone based applications increased

-Fitbit, polar, consumer and research grade accelerometers use increased - Validity and reliability low

- Reduction in daily step count (-2812 steps)

- Reduction in active time $(-30 \%)$

- Reduced physical activity is adversely

associated with stress and autonomic responses

interface provide more reliable and easily understandable data visualization, thereby gaining popularity, which is seen in the included studies of our review [28]. Smartwatches, smart bands, smart rings, and bracelets are widespread in human activity monitoring and behavior change [36]. Furthermore, wearables embedded with behavior change techniques such as goal setting, information/counseling, prompts, motivation and social support make wearables a potential choice for increased compliance to behavior interventions and long-term behavior change. We found that the behavioral techniques are least addressed in the studies that have employed the wearables for improving physical activity or reducing sedentary behavior in individuals during the pandemic $[21,24]$. We recommend that future wearables be developed along with behavioral scientists to understand target behavior (intensity and type of activity) and incorporate maximum behavior change techniques for higher compliance in community settings [37].

\section{Physical health regulation with wearables during COVID-19}

Our review results showed active time in all age groups is significantly reduced by at least $30-50 \%$ after lockdown 
compared to pre-pandemic periods $[16,17,19,21,22,25$, 26]. Our findings concur with the recent cross-sectional study that investigated smartphone-based physical activity measurement before and after lockdown and found $37 \%$ reduction in weekly minutes of PA [38]. Consumer-based wearables allowed users to monitor their activity levels and may potentially improve their compliance toward longterm behavior change, which are increasingly popular during this pandemic. Brickwood et al. (2019) systematically reviewed 28 randomized controlled trials and found a significant increase in step count (standardized mean difference [SMD] $0.24 ; 95 \%$ CI 0.16-0.33), moderate-to-vigorous physical activity (SMD 0.27 ; 95\% CI 0.15-0.39) and energy expenditure (SMD 0.28; 95\% CI 0.03-0.54) with wearables use [39]. Our review findings concur with the results of the above review, which concluded that physical activity and active time could be improved significantly with wearable devices. In our review, most of the included studies involved healthy adults and four studies investigated physical activity changes in pregnant women [27], asthmatic children [25] and diabetes population $[18,24]$. We could also find a trend of studies exploring the use of wearable technology in the elderly for improving self-efficacy and behavioral change for physical activity promotion [24, 26].

Restriction on sports amenities, public gathering, home confinement and remote work are some of the potential barriers to adequate physical activity practises during this pandemic [40]. We propose wearables to be an intriguing intervention for measuring, advocating and monitoring physical activity for reaping health benefits for being active during this pandemic [41]. Furthermore, the reduction of active time was adversely associated with physical and mental health [42]. The physiological changes such as improved cortisol, a brain-derived neurotrophic factor associated with increased activity time, are postulated to have favorable effects on mental health [43, 44]. Nevertheless, a longitudinal study by Vile et al. [27] found a reduction in the heart rate variability with lower physical activity during lockdown might have unfavorable effects on cardiovascular disease risk. Thus, wearable technology serves as an intriguing means to promote physical activity and reduce sedentary behavior among healthy population and individuals at risk for chronic diseases during these unprecedented times.

\section{Limitations}

Few limitations are worth mentioning: (1) majority of the articles included were conducted between early lockdown (March 2020-June 2020). Hence, the review findings may not represent the intensity of wearables use and their impact on physical activity at present; (2) we observed heterogeneity in terms of age, wearable make and working mechanisms of the Internet of Things and algorithms associated with the cloud transfer and interpretation; (3) furthermore, confounding factors such as financial incentives, personalized coaching, wear time, and multifaceted intervention might be the barriers to establishing the effectiveness of wearables in physical activity advocacy. Hence, our review findings should be interpreted with caution for generalisability; (4) as a scoping review, we aimed to provide an overview or map the existing evidence rather than critically appraising answer to a particular question. Therefore, policy makers should consider the breadth of the existing knowledge rather than depth and provide framework for public health practises when interpreting our review findings. We recommend that future systematic reviews summarize evidence of the impact of wearables on public health that can be translated from research into practice.

\section{Conclusion}

Wearables appear to be impending means of improving physical activity and reducing sedentary behavior remotely during this pandemic. National and organizational policies should adopt wearable technologies to promote physical activity, thereby reducing chronic diseases in the general population.

Supplementary Information The online version contains supplementary material available at https://doi.org/10.1007/s11332-021-00885-x.

Acknowledgements Authors wish to thank Dr Fiddy Davis, Head of the department, Department of Exercise and Sports Sciences, Manipal College of Health Professions, Manipal Academy of Higher Education, Karnataka, India for his support towards research.

Author contributions $\mathrm{BC}$ conceived and designed review protocol. BC and RP conducted the search and analyzed the studies that investigated the wearables on physical health during COVID-19 pandemic. BC wrote the original draft of the manuscript and RP edited the manuscript. Both the authors read and approved the final version of manuscript.

Funding Open access funding provided by Manipal Academy of Higher Education, Manipal.

Data availability All available data have been presented in the study.

Code availability Not applicable.

\section{Declarations}

Conflicts of interest None of the authors has any conflicts of interest.

Ethical approval Not applicable.

Consent to participate Not applicable.

Consent for publication Not applicable. 
Open Access This article is licensed under a Creative Commons Attribution 4.0 International License, which permits use, sharing, adaptation, distribution and reproduction in any medium or format, as long as you give appropriate credit to the original author(s) and the source, provide a link to the Creative Commons licence, and indicate if changes were made. The images or other third party material in this article are included in the article's Creative Commons licence, unless indicated otherwise in a credit line to the material. If material is not included in the article's Creative Commons licence and your intended use is not permitted by statutory regulation or exceeds the permitted use, you will need to obtain permission directly from the copyright holder. To view a copy of this licence, visit http://creativecommons.org/licenses/by/4.0/.

\section{References}

1. Wang C, Tee M, Roy AE, Fardin MA, Srichokchatchawan W, Habib HA et al (2021) The impact of COVID-19 pandemic on physical and mental health of Asians: a study of seven middleincome countries in Asia. PLoS ONE 16(2):e0246824. https://doi. org/10.1371/journal.pone.0246824

2. Curtis RG, Olds T, Ferguson T, Fraysse F, Dumuid D, Esterman A et al (2021) Changes in diet, activity, weight, and wellbeing of parents during COVID-19 lockdown. PLoS ONE 16(3):e0248008. https://doi.org/10.1371/journal.pone.0248008

3. Oakman J, Kinsman N, Stuckey R, Graham M, Weale V (2020) A rapid review of mental and physical health effects of working at home: how do we optimise health? BMC Public Health 20(1):1825. https://doi.org/10.1186/s12889-020-09875-z

4. Thivel D, Tremblay A, Genin PM, Panahi S, Rivière D, Duclos M (2018) Physical activity, inactivity, and sedentary behaviors: definitions and implications in occupational health. Front Public Health 6:288. https://doi.org/10.3389/fpubh.2018.00288

5. González K, Fuentes J, Márquez JL (2017) Physical inactivity, sedentary behavior and chronic diseases. Korean J Fam Med 38(3):111-115. https://doi.org/10.4082/kjfm.2017.38.3.111

6. Booth FW, Roberts CK, Laye MJ (2012) Lack of exercise is a major cause of chronic diseases. Compr Physiol 2(2):1143-1211. https://doi.org/10.1002/cphy.c110025

7. Hall G, Laddu DR, Phillips SA, Lavie CJ, Arena R (2021) A tale of two pandemics: how will COVID-19 and global trends in physical inactivity and sedentary behavior affect one another? Prog Cardiovasc Dis 64:108-110. https://doi.org/10.1016/j.pcad. 2020.04.005

8. Silva DR, Werneck AO, Malta DC, Souza-Júnior PRB, Azevedo LO, Barros MBA et al (2021) Incidence of physical inactivity and excessive screen time during the first wave of the COVID-19 pandemic in Brazil: what are the most affected population groups?: impact of COVID-19 pandemic on physical activity and screen time in Brazil. Ann Epidemiol 62:30-35. https://doi.org/10.1016/j. annepidem.2021.05.001

9. Wilde LJ, Ward G, Sewell L, Müller AM, Wark PA (2018) Apps and wearables for monitoring physical activity and sedentary behaviour: a qualitative systematic review protocol on barriers and facilitators. Digit Health 4:2055207618776454. https://doi. org/10.1177/2055207618776454

10. Research GV (2020) Wearable technology (2020-2027). Wearable technology market size, share and trends analysis report by product (wrist-wear, eye-wear \& head-wear, foot-wear, neck-wear, body-wear), by application, by region, and segment forecasts, 2020-2027. https://www.grandviewresearch.com/industry-analy sis/wearable-technology-market. Accessed 20 Nov 2021
11. Weizman Y, Tan AM, Fuss FK (2020) Use of wearable technology to enhance response to the Coronavirus (COVID-19) pandemic. Public Health 185:221-222. https://doi.org/10.1016/j.puhe.2020. 06.048

12. O'Keeffe N, Scheid JL, West SL (2020) Sedentary behavior and the use of wearable technology: an editorial. Int J Environ Res Public Health 17(12):4181. https://doi.org/10.3390/ijerph1712 4181

13. Mercer K, Li M, Giangregorio L, Burns C, Grindrod K (2016) Behavior change techniques present in wearable activity trackers: a critical analysis. JMIR Mhealth Uhealth 4(2):e40. https://doi. org/10.2196/mhealth.4461

14. Levac D, Colquhoun H, O'Brien K (2010) Scoping studies: advancing the methodology. Implement Sci 5:69. https://doi.org/ 10.1186/1748-5908-5-69

15. Arksey H, O'Malley L (2005) Scoping studies: towards a methodological framework. Int J Soc Res Methodol 8(1):19-32. https:// doi.org/10.1080/1364557032000119616

16. Ammar A, Bouaziz B, Trabelsi K, Glenn JM, Zmijewski P, Müller $\mathrm{P}$ et al (2021) Applying digital technology to promote active and healthy confinement lifestyle during pandemics in the elderly. Biol Sport 38(3):391-396. https://doi.org/10.5114/biolsport.2021. 100149

17. Ammar A, Trabelsi K, Brach M, Chtourou H, Boukhris O, Masmoudi L et al (2021) Effects of home confinement on mental health and lifestyle behaviours during the COVID-19 outbreak: insights from the ECLB-COVID19 multicentre study. Biol Sport 38(1):9-21. https://doi.org/10.5114/biolsport.2020.96857

18. Ang IYH, Tan KXQ, Tan C, Tan CH, Kwek JWM, Tay J et al (2021) A personalized mobile health program for type 2 diabetes during the COVID-19 pandemic: single-group pre-post study. JMIR Diabetes 6(3):e25820. https://doi.org/10.2196/25820

19. Buoite Stella A, Ajcevic M, Furlanis G, Cillotto T, Menichelli A, Accardo A et al (2021) Smart technology for physical activity and health assessment during COVID-19 lockdown. J Sports Med Phys Fitness 61(3):452-460. https://doi.org/10.23736/s00224707.20.11373-2

20. Capodilupo ER, Miller DJ (2021) Changes in health promoting behavior during COVID-19 physical distancing: utilizing wearable technology to examine trends in sleep, activity, and cardiovascular indicators of health. PLoS ONE 16(8):e0256063. https:// doi.org/10.1371/journal.pone.0256063

21. Ding D, Cheng M, del Pozo CB, Lin T, Sun S, Zhang L et al (2021) How COVID-19 lockdown and reopening affected daily steps: evidence based on 164,630 person-days of prospectively collected data from Shanghai, China. Int J Behav Nutr Phys Act 18(1):1-10. https://doi.org/10.1186/s12966-021-01106-x

22. Hamasaki H (2021) Daily physical activity and sleep measured by wearable activity trackers during the coronavirus disease 2019 pandemic: a lesson for preventing physical inactivity during future pandemics. Appl Sci (Switzerland) 11(21):9956. https://doi.org/ 10.3390/app11219956

23. Henriksen A, Johannessen E, Hartvigsen G, Grimsgaard S, Hopstock LA (2021) Consumer-based activity trackers as a tool for physical activity monitoring in epidemiological studies during the COVID-19 pandemic: development and usability study. JMIR Public Health Surveill 7(4):e23806. https://doi.org/10.2196/23806

24. Jiwani R, Dennis B, Bess C, Monk S, Meyer K, Wang J et al (2021) Assessing acceptability and patient experience of a behavioral lifestyle intervention using fitbit technology in older adults to manage type 2 diabetes amid COVID-19 pandemic: a focus group study: focus group analysis of lifestyle intervention. Geriatr Nurs 42(1):57-64. https://doi.org/10.1016/j.gerinurse.2020.11.007 
25. Kouis P, Michanikou A, Anagnostopoulou P, Galanakis E, Michaelidou E, Dimitriou H et al (2021) Use of wearable sensors to assess compliance of asthmatic children in response to lockdown measures for the COVID-19 epidemic. Sci Rep 11(1):5895. https://doi.org/10.1038/s41598-021-85358-4

26. Mishra R, Park C, York MK, Kunik ME, Wung SF, Naik AD et al (2021) Decrease in mobility during the COVID-19 pandemic and its association with increase in depression among older adults: a longitudinal remote mobility monitoring using a wearable sensor. Sensors 21(9):3090. https://doi.org/10.3390/s21093090

27. Niela-Vilén H, Auxier J, Ekholm E, Sarhaddi F, Asgari Mehrabadi M, Mahmoudzadeh A et al (2021) Pregnant women's daily patterns of well-being before and during the COVID-19 pandemic in Finland: longitudinal monitoring through smartwatch technology. PLoS ONE 16(2):e0246494. https://doi.org/10.1371/journal.pone. 0246494

28. Pepin JL, Bruno RM, Yang RY, Vercamer V, Jouhaud P, Escourrou $\mathrm{P}$ et al (2020) Wearable activity trackers for monitoring adherence to home confinement during the COVID-19 pandemic worldwide: data aggregation and analysis. J Med Internet Res 22(6):e19787. https://doi.org/10.2196/19787

29. Speirs C, Granat M, Stamatakis E, Hamer M (2021) Estimating changes in physical behavior during lockdowns using accelerometry-based simulations in a large UK cohort. Scand J Med Sci Sports 31(12):2221-2229. https://doi.org/10.1111/sms.14032

30. Sañudo B, Fennell C, Sánchez-Oliver AJ (2020) Objectivelyassessed physical activity, sedentary behavior, smartphone use, and sleep patterns pre- and during-COVID-19 quarantine in young adults from Spain. Sustainability 12(15):5890

31. Wang Y, Zhang Y, Bennell K, White DK, Wei J, Wu Z et al (2020) Physical distancing measures and walking activity in middle-aged and older residents in Changsha, China, during the COVID-19 epidemic period: longitudinal observational study. J Med Internet Res 22(10):e21632. https://doi.org/10.2196/21632

32. Woodruff SJ, Coyne P, St-Pierre E (2021) Stress, physical activity, and screen-related sedentary behaviour within the first month of the COVID-19 pandemic. Appl Psychol Health Well Being 13(2):454-468. https://doi.org/10.1111/aphw.12261

33. Greco L, Percannella G, Ritrovato P, Tortorella F, Vento M (2020) Trends in IoT based solutions for health care: moving AI to the edge. Pattern Recognit Lett 135:346-353. https://doi.org/10. 1016/j.patrec.2020.05.016

34. Strain T, Wijndaele K, Dempsey PC, Sharp SJ, Pearce M, Jeon $\mathrm{J}$ et al (2020) Wearable-device-measured physical activity and future health risk. Nat Med 26(9):1385-1391. https://doi.org/10. 1038/s41591-020-1012-3

35. Cvetkovic B, Szeklicki R, Janko V, Lutomski P, Lustrek M (2018) Real-time activity monitoring with a wristband and a smartphone. Inf Fusion 43:77-93. https://doi.org/10.1016/j.inffus.2017.05.004
36. Henriksen A, Haugen Mikalsen M, Woldaregay AZ, Muzny M, Hartvigsen G, Hopstock LA et al (2018) Using fitness trackers and smartwatches to measure physical activity in research: analysis of consumer wrist-worn wearables. J Med Internet Res 20(3):e110. https://doi.org/10.2196/jmir.9157

37. Chandrasekaran B, Kundapur PP, Rao CR (2021) Are free workplace health promotion apps adequately mapped with behavior change theories, techniques and desired features? A content analysis. Transl Behav Med 11(8):1507-1516. https://doi.org/10.1093/ tbm/ibab042

38. McCarthy H, Potts HWW, Fisher A (2021) Physical activity behavior before, during, and after COVID-19 restrictions: longitudinal smartphone-tracking study of adults in the United Kingdom. J Med Internet Res 23(2):e23701. https://doi.org/10.2196/23701

39. Brickwood KJ, Watson G, O'Brien J, Williams AD (2019) Consumer-based wearable activity trackers increase physical activity participation: systematic review and meta-analysis. JMIR Mhealth Uhealth 7(4):e11819. https://doi.org/10.2196/11819

40. Stockwell S, Trott M, Tully M, Shin J, Barnett Y, Butler L et al (2021) Changes in physical activity and sedentary behaviours from before to during the COVID-19 pandemic lockdown: a systematic review. BMJ Open Sport Exerc Med 7(1):e000960. https:// doi.org/10.1136/bmjsem-2020-000960

41. McCallum C, Rooksby J, Gray CM (2018) Evaluating the impact of physical activity apps and wearables: interdisciplinary review. JMIR Mhealth Uhealth 6(3):e58. https://doi.org/10.2196/mheal th.9054

42. Meyer J, McDowell C, Lansing J, Brower C, Smith L, Tully M et al (2020) Changes in physical activity and sedentary behavior in response to COVID-19 and their associations with mental health in 3052 US adults. Int J Environ Res Public Health 17(18):e6469. https://doi.org/10.3390/ijerph17186469

43. Chandrasekaran B, Pesola AJ, Rao CR, Arumugam A (2021) Does breaking up prolonged sitting improve cognitive functions in sedentary adults? A mapping review and hypothesis formulation on the potential physiological mechanisms. BMC Musculoskelet Disord 22(1):274. https://doi.org/10.1186/s12891-021-04136-5

44. Wennberg P, Boraxbekk CJ, Wheeler M, Howard B, Dempsey PC, Lambert G et al (2016) Acute effects of breaking up prolonged sitting on fatigue and cognition: a pilot study. BMJ Open 6(2):e009630. https://doi.org/10.1136/bmjopen-2015-009630

Publisher's Note Springer Nature remains neutral with regard to jurisdictional claims in published maps and institutional affiliations. 